\title{
Adherencia a las inmunizaciones en niños nacidos con menos de 1.500 gr de peso o antes de 32 semanas de gestación, en dos centros chilenos
}

\author{
Gonzalo Calderón C., Rosario Moore V., Enrica Pittaluga P. y Marcela Potin S.
}

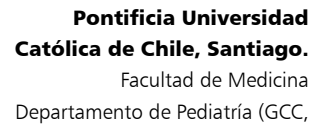

RMV, MPS).

Complejo Asistencial Dr. Sótero del Río, Santiago. Servicio de Neonatología. (EPP).

Recibido: 22 de marzo de 2010 Aceptado: 01 de enero de 2011

Correspondencia a: Gonzalo Calderón Cabrera gonzaloc82@hotmail.com

\section{Adherence to immunizations in newborns less than $1500 \mathrm{gr}$ at birth and/or younger than 32 weeks, in two chilean centers}

Preterm neonates less than 1500 gr. or younger than 32 weeks have an increased morbidity and mortality due to infectious diseases. Immunization of these children is critical but is often incomplete and delayed. Objectives: To describe the adherence of scheduled and additional vaccines recommended in preterms less than 1500 gr. and to compare the level of compliance in two centers. Patients and Methods: Prematures less than 1500 gr. born between January 2006 and December 2008 in a private center (HCPUC) and in a public health system (HSR) were included. Neonatal charts and follow up records were reviewed. Demographic data, diagnosis, scheduled vaccines, delays and its causes, prescription of additional vaccines were registered. Results: 92 children were included in HCPUC and 246 in HSR. Among these $60-70 \%$ respectively had delayed immunizations. The reasons for these were considered not justified in almost $80 \%$ of the cases, being the primary reason oversight or lack of parental time. The use of additional vaccines is still low in both centers. Conclusions: Vaccination of prematures in Chile requires important improvements; education of healthcare personnel and parents, inclusion of modern computerized records and inclusion of new efficacious and safe vaccines in the regular immunization program with no cost to the patient.

Key words: Vaccines, preterm babies, immunization, adherence.

Palabras clave: Vacunas, prematuros, inmunizaciones, adherencia.

\section{Introducción}

$\mathrm{E}$ 1 uso sistemático de vacunas es una medida de gran impacto en salud pública ${ }^{1}$. Se estima que la práctica obligatoria de vacunación en niños sanos a través del Programa Nacional de Inmunizaciones (PNI), previene tres millones de muertes anualmente en el mundo ${ }^{2}$. Sin embargo, a pesar de la disponibilidad de múltiples vacunas eficaces y seguras, su uso es aún muy limitado en grupos especiales como los niños prematu$\operatorname{ros}^{3-4}$. Estos pacientes son particularmente susceptibles a infecciones bacterianas y virales por diversas razones. Algunas de las infecciones inmunoprevenibles que pueden afectar al niño prematuro son frecuentes y graves, como ha sido expresado en la literatura científica. Tres ejemplos de ello son:

- El riesgo de enfermedad por Bordetella pertussis es casi el doble que el de niños de término (RR 1,86, IC $95 \% 1,33-2,38)^{5}$.

- Para enfermedad neumocóccica invasora, el riesgo en prematuros de menos de 1.500 gr o 32 semanas es entre 6,7 y 9,1 veces mayor que en niños de término ${ }^{6}$.

- Para influenza el problema es también notable pues hasta $10 \%$ de las muertes por este agente en niños ocurren en prematuros con patología cardíaca, respiratoria o renal asociada ${ }^{7,8}$.

La prevención de infecciones a través del uso de vacunas, debiera ser un eje en el cuidado de estos niños; no obstante, los estudios demuestran, con frecuencia, atrasos o esquemas incompletos de vacunación en comparación con el niño de término ${ }^{9-11}$. A la fecha de este estudio, el esquema de vacunación PNI para niños prematuros en nuestro país incluía las siguientes vacunas:

- BCG: en niños que han alcanzado más de 2.000 gr.

- Difteria, tétanos, tos ferina (coqueluche) (DTP), Haemophilus influenzae tipo b, hepatitis B y polio oral trivalente (VPO) a los 2, 4 y 6 meses de vida.

- DTP y VPO a los 18 meses.

- Sarampión, parotiditis y rubeóla (vacuna tresvírica) a los 12 meses.

- Vacuna anti-influenza en niños sobre 6 meses de edad, en forma anual, con un esquema de dos dosis el primer año de vacunación y una dosis las temporadas siguientes. 
- Vacuna neumocóccica conjugada heptavalente (PCV7) a los 2, 4 y 6 meses, con un refuerzo entre los 12 meses y 15 meses de edad. Esta vacuna inicialmente estaba disponible sólo para niños con displasia broncopulmonar y en el año 2008 se amplió la indicación a todos los niños prematuros.

Como referencia, se adjuntan en la Figura 1 las recomendaciones adaptadas de la Academia Americana de Pediatría a nuestro medio y que para efectos de este estudio se definieron como la vacunación ideal del prematuros ${ }^{12-17}$.

La literatura internacional muestra que este esquema difícilmente se cumple en prematuros de muy bajo peso y que las mayoría de las desviaciones del esquema de inmunización se concentran en los primeros 6 meses de vida $^{18}$. Esto podría ser explicado por contraindicaciones temporales de las vacunas, infecciones intercurrentes, enfermedades asociadas a la prematurez o a que una proporción de los prematuros de muy bajo peso está aún hospitalizado cuando deben iniciar su vacunación. Sin embargo, hay evidencia de que los retrasos en la indicación de las vacunas rutinarias con frecuencia no son explicables, al menos, por la persistencia de síntomas de enfermedades crónicas propias del prematuro ${ }^{18}$.

Otro factor que puede influir en el menor cumplimiento es el temor o desconocimiento sobre la seguridad de las vacunas en estos niños. La información sobre este aspecto en prematuros, y en especial en recién nacidos de muy bajo peso de nacimiento (RNMBPN), es muy escasa.

En general, la administración de vacunas es segura en niños estables, aunque se describe mayor frecuencia de episodios de apnea y desaturación de oxígeno posterior a la vacunación, probablemente como una respuesta inespecífica al estrés del niño prematuro ${ }^{19}$.

Si bien la información sobre inmunogenicidad es limitada, los datos disponibles sugieren que estos niños responden con niveles protectores de anticuerpos para todos lo antígenos desde el mes de vida en adelante, por lo que deben ser vacunados según edad cronológica y no según edad corregida ${ }^{20}$.

Respecto a la persistencia de anticuerpos en el tiempo, la información demuestra que, aunque se detecta memoria inmunológica, los niveles de anticuerpos declinan más rápidamente por lo que el uso de dosis de refuerzo, así como el de un esquema completo es aún más necesario que en niños de término ${ }^{21}$.

Existe escasa información publicada en nuestro país sobre el cumplimiento del programa de inmunizaciones y el uso de vacunas adicionales en niños prematuros y en especial en RNMBPN. Objetivo: Realizar un estudio descriptivo de la adherencia a la vacunación en RNMBPN, comparando las coberturas de vacunación en dos centros de la Región Metropolitana, uno privado y otro estatal, donde se mantiene un seguimiento sistemático de estos niños.

\section{Pacientes y Método}

Diseño: Estudio observacional.

Pacientes: Se revisaron las bases de datos que registra a todos los prematuros menores de 32 semanas de gestación y/o peso de nacimiento menor a $1.500 \mathrm{gr}$, ingresados a los Servicios de Neonatología del Hospital Dr. Sótero del Río (HSR) y al Hospital Clínico de la Pontificia Universidad Católica de Chile (HCPUC), en el período comprendido entre el 1 de enero de 2006 y el 31 de diciembre de 2008. Fueron excluidos los prematuros fallecidos durante la estadía en Neonatología o posteriormente, así como pacientes sin controles posteriores al alta en el centro respectivo y aquellos sin un registro escrito disponible de las vacunas administradas.

\begin{tabular}{|c|c|}
\hline \multicolumn{2}{|c|}{$\begin{array}{l}\text { Academia Americana de Pediatría } 2003 . \\
\text { Advisory Committee on Immunization Practices } 2005 \text { \& } 2006 \text {. }\end{array}$} \\
\hline Vacuna(s) & Edad y esquemas de vacunación \\
\hline $\mathrm{BCG}^{1}$ & Al nacer. \\
\hline Anti-Hib DTP22 & $2,4,6$ meses - refuerzo 18 meses de vida. \\
\hline Anti- hepatitis $B^{3}$ & $0-1,2-3,6-7$ meses o esquemas 2,4 y 6 meses \\
\hline Anti-polio & $2,4,6$ y 18 meses. \\
\hline Tres vírica & Una dosis 12 meses \\
\hline Anti varicela ${ }^{4}$ & $\begin{array}{l}2 \text { dosis: } 1^{\text {a }} \text { dosis } 12 \text { meses - } 15 \text { meses } \\
2^{\text {a }} \text { dosis entre los } 4 \text { y } 6 \text { años. }\end{array}$ \\
\hline Anti-influenza & Anual desde los 6 meses de vida \\
\hline Anti-neumocóccica conjugada & $2,4,6$ meses - refuerzo 12 a 15 meses \\
\hline Anti-hepatitis $A^{5}$ & Dos dosis a partir de 2 años esquema 0 y 6 meses \\
\hline Anti-rotavirus & $\begin{array}{l}\text { Esquema } 3 \text { dosis, 2, } 4 \text { y } 6 \text { meses }^{6} \text {. } \\
\text { Esquema } 2 \text { dosis } 2 \text { y } 4 \text { meses }^{7} \text {. }\end{array}$ \\
\hline
\end{tabular}

'Bacilo de Calmette Guerin: 80\% de los países del mundo utiliza BCG al nacer. La recomendación es administrar la vacuna sólo después que los niños han alcanzado más de $2.000 \mathrm{~g}$ Para esta recomendación, sólo existe evidencia tipo E (opinión de expertos).

2En Chile se utilizan vacunas anti-pertussis de células enteras. Para el refuerzo en el segundo año de vida se utiliza actualmente año 2010 vacuna DTP-HB/Hib En E.U.A y algunos países europeos se utilizan vacunas anti-pertussis acelulares.

${ }^{3}$ Los prematuros de menos de $2.000 \mathrm{~g}$ requieren ajustar el esquema de inmunización dependiendo de la medición de antígeno de superficie ( $\mathrm{HBsAg}$ ) en la madre. Si la madre es $\mathrm{HBsAg}(+)$ o de condición desconocida, se debe usar 1 dosis de vacuna al nacer e inmunoglobulina hiperinmune ( $\mathrm{HBIG}$ ) antes de las 12 horas de vida, en sitios de inyección diferentes. Si la madre es HBsAg (-) se debe administrar la primera dosis al mes o a los 2 meses de vida, ya que la respuesta inmune es superior con este esquema.

${ }^{4}$ La Academia Americana de Pediatría desde el 2007 recomienda una segunda dosis de vacuna antivaricela antes de la entrada del niño a jardín infantil o primero básico.

${ }^{5} \mathrm{E}$ El esquema de vacunación anti-hepatitis A incluye 2 dosis separadas por 6-12 meses. En Chile está registrada para su uso a partir de los 24 meses de vida pero hay datos suficientes para su uso en niños sobre un año de edad.

${ }^{6}$ Esquema de la vacuna Rotateq ${ }^{\circledR}$ MSD, aplicable a niños mayores de 6 semanas de vida. ${ }^{7}$ Esquema de la vacuna Rotarix® GSK, aplicable a niños mayores de 6 semanas de vida.

Figura 1. Esquema adaptado de la recomendación de la Academia Americana de Pediatría para la vacunación del prematuro. 
Los prematuros nacidos en el HCPUC y en el HSR fueron controlados en dichos centros; sin embargo, las vacunas en niños del HSR fueron administradas en los distintos consultorios del área metropolitana sur-oriente, a los cuales pertenecían, excepto la vacuna PCV7, la cual se administró en el mismo HSR.

Uno de los centros analizados, (HCPUC), atiende pacientes con sistemas previsionales privado y estatal, 55 y $45 \%$ respectivamente, recibe pacientes de diversas comunas de Santiago, y registra 2.400 partos al año; $1,7 \%$ son niños con menos de 1.500 gr de peso al nacer ${ }^{22}$. El otro centro, (HSR), atiende sólo pacientes del sistema previsional estatal, fundamentalmente del sector sur-oriente de la Región Metropolitana, registrando 7.300 partos al año, de los cuales $1,8 \%$ corresponden a neonatos con peso inferior a $1.500 \mathrm{gr}$ al nacer ${ }^{23}$.

Información recolectada: Se registró a partir de las fichas clínicas los siguientes datos: fecha y peso de nacimiento, edad gestacional, patología neonatal (displasia broncopulmonar, hemorragia intracerebral, encefalopatía hipóxico-isquémica, enterocolitis necrosante, cardiopatías congénitas y síndromes genéticos (distintos a síndrome Down), hospitalizaciones posteriores al alta, edad de la madre, existencia de familia monoparental.

Los datos de vacunación fueron obtenidos exclusivamente de registros escritos (carné de inmunizaciones, cuaderno de control pediátrico o ficha clínica). El resto de la información se obtuvo mediante encuesta telefónica con los cuidadores del niño, a los que se les pidió verificar su carné de vacunación para responder. Se les preguntó por razones de los atrasos si existían, recomendación de vacunas no PNI por parte del médico tratante, así como su administración efectiva. Para evaluar las razones para la no administración de una determinada vacuna o de su atraso, se realizó una pregunta abierta y se les pidió mencionar el principal motivo, considerándose sólo éste para la tabulación de datos.

Sólo se registraron las vacunas administradas durante los primeros tres años de vida.

En el caso de niños bajo tres años de edad se consideró el esquema de vacunación hasta el momento de la recolección de datos.

Definiciones. Para efectos de este estudio se usaron las siguientes definiciones:

- Atraso de inmunización PNI: administración de una vacuna PNI después de 30 días de la edad recomendada o del intervalo de tiempo sugerido entre una y otra dosis ${ }^{24-25}$.

- Atraso de inmunización justificado: atraso explicado por hospitalización, morbilidad aguda significativa, reacción adversa grave a dosis previa, contraindicación por enfermedad de base descrita en la literatura. Esta definición sólo se aplicó a las vacunas PNI.
- Atraso de inmunización no justificado: atraso debido a morbilidad menor (infección respiratoria alta $\mathrm{y} / \mathrm{o}$ baja, sin requerimientos de oxígeno o de hospitalización, síndrome diarreico agudo sin deshidratación, exantema sin fiebre), o falta de vacunación asociada a olvidos, falta de tiempo por parte de los padres, lejanía del lugar de administración y contraindicaciones no documentadas en la literatura. Esta definición sólo se aplicó a vacunas PNI.

- Vacunas no PNI: vacunas contra la hepatitis A, varicela, rotavirus, Streptococcus pneumoniae polisacárida de 23 serotipos ( $\mathrm{PV} 23$ ), anti-meningoccócica $\mathrm{C}$ conjugada.

- Vacunación óptima PNI: Esquema PNI completo y $\sin$ atraso; adicionalmente uso de vacuna antineumocóccica conjugada heptavalente (PCV7), con el número de dosis completas para la edad y sin atrasos.

- Vacunación ideal del prematuro: Esquema PNI completo y sin atrasos; adicionalmente uso de las siguientes vacunas: anti-neumocóccica conjugada heptavalente (PCV7), anti-rotavirus, anti-varicela, anti-hepatitis A, con el número de dosis completas para la edad y sin atrasos.

Análisis estadístico. Las variables categóricas fueron descritas en términos de número y frecuencia y las variables numéricas en términos de promedio y desviación estándar. Las asociaciones entre variables categóricas se evaluaron utilizando la prueba de chi-cuadrado, mientras que las asociaciones entre variables categóricas y numéricas fueron evaluadas en base a la prueba de MannWhitney. Se consideró estadísticamente significativo todo valor $\mathrm{p}<0,05$. Los cálculos fueron realizados utilizando el programa SPSS 15.0

\section{Resultados}

En el HCPUC la base de datos registró 129 RNMBPN ingresados entre enero de 2006 y diciembre de 2008; 92 $(71,3 \%)$ de ellos fueron incluidos en el estudio. La diferencia (37 niños) correspondió a 10 fallecidos, 23 traslados a otros hospitales, dos sin registro escrito de vacunas y dos niños no ubicados por los autores. En el HSR se registraron 348 pacientes, y se incluyeron 246 (70,7\%). La diferencia (102 niños) correspondió a 11 fallecidos, 20 traslados a otros hospitales y 71 niños no ubicables para la encuesta telefónica y seguimiento en policlínico.

Las Tablas 1 y 2 resumen los datos bio-demográficos de ambos centros. Sólo se observan diferencias para la edad materna (15\% madres adolescentes en el HSR) y para la proporción de niños con cardiopatía congénita en el HCPUC.

La frecuencia de administración oportuna de todas las vacunas PNI en el HCPUC fue de 40\% (37/92), y en el 
HSR fue de $30 \%$ (74/246), con un valor p de 0,08. En los niños con esquema incompleto, $55 \%$ no recibió una (o más) dosis de vacuna y $45 \%$ la recibió pero con atraso. Esto fue similar en ambos centros (p 0,09).

El cumplimiento de la vacunación con BCG fue de $100 \%$ en ambos hospitales; la adherencia a las otras vacunas se observa en la Tabla 3 . En el grupo de niños hospitalizados por más de 60 días no se registraron atrasos en la primera dosis de DTP-HB/Hib en $77,5 \%(31 / 40)$ en el HCPUC y $85,8 \%(73 / 85)$ de los niños en el HSR $(\mathrm{p}=0,83)$.

Las razones más frecuentemente referidas por los padres para el atraso o no administración de las vacunas fueron: hospitalización por intercurrencias $(29 \%$ en el HPUC y $47 \%$ en el HSR, $p=0,018)$ y olvidos o falta de tiempo de los padres (35\% en el HPUC y $42 \%$ en HSR, $\mathrm{p}=0,33)$. Los padres no reportaron reacciones adversas a dosis previas como causa en ninguno de los centros y sólo se consignó contraindicación por patología de base en $1 \%$ de los niños en el HSR y ninguno en el HCPUC. En una pequeña proporción de niños, la razón de atraso fue la indicación de vacunar según edad corregida y no cronológica, $3 \%$ en el HCPUC y $9 \%$ en el HSR (p 0,04). Al analizar detalladamente todas las causas para los atrasos de vacunación, se concluyó que el 78\% de ellas no fueron realmente justificadas según las definiciones operativas de este trabajo.

Las coberturas de inmunización anti-influenza se observan en la Figura 2. Cerca de $90 \%$ de los niños recibe la primera dosis de esta vacuna pero esto no se mantiene con la segunda dosis, en especial en temporadas sucesivas de influenza. Este hecho fue similar en ambos centros $(\mathrm{p}>0,05)$.

En la Tabla 4 se observa el análisis de algunos factores de riesgo con diferencia estadísticamente significativa para una menor adherencia a las vacunas. Destaca el peso menor a $1.000 \mathrm{gr}$, edad gestacional menor de 27 semanas, presencia de alguna patología del prematuro y hospitalizaciones posteriores al alta de Neonatología.

En relación a la vacuna $\mathrm{PCV} 7$ se administró al menos una dosis a $68 \%(63 / 92)$ de los prematuros en el HCPUC y a 74\% (182/246) en el HSR (p: NS). El esquema completo de esta vacuna ajustado por edad al momento de la encuesta, sólo se administró a $15,2 \%$ de los niños en HCPUC y a 9,3\% en el HSR (p: 0,12). Las razones más frecuentemente referidas por los padres para no recibir la vacuna fueron, en el HCPUC el factor económico (58\%) y omisión de la indicación del pediatra (19\%) y en el HSR las dos principales razones fueron omisión del pediatra $(57,3 \%)$ y falta de disponibilidad de dosis de vacuna al momento de la indicación $(30,5 \%)$.

Hubo diferencias estadísticamente significativas en el uso de otras vacunas extra PNI; 51\% (47/92) de los niños en el HCPUC recibieron recomendación de éstas, y 9\% de los niños $(22 / 246)$ en el HSR $(p<0,01)$. Las vacunas más recomendadas en el HCPUC fueron la anti-varicela y la anti-hepatitis A (42 y $41 \%$, respectivamente), mientras que en el HSR lo fue la vacuna anti-neumocóccica polisacárida de 23 serotipos en $46 \%$ de los niños. La administración efectiva de las vacunas recomendadas fue de $53 \%$ en el HCPUC y $58 \%$ en el HSR (p: NS).

Con respecto a la definición de vacunación óptima PNI en el HCPUC, sólo se cumplió en 5\% (5/92) en el HCPUC y 4\% (9/246) en HSR, $(p=0,67)$.

Ningún niño cumplió con la definición de vacunación ideal del prematuro y esto fue idéntico en ambos centros.

Tabla 1. Características bio-demográficas de prematuros de muy bajo peso en dos centros

\begin{tabular}{lccc} 
Variables & HCPUC & HSR & Valor p \\
& $\mathrm{n}: 92$ & $\mathrm{n}: 246$ & \\
Género masculino (\%) & $50 \%$ & $52 \%$ & 0,69 \\
Peso promedio al nacer (gr) & $1.236 \pm 370$ & $1.303 \pm 352$ & 0,11 \\
Niños $\leq 1.000 \mathrm{gr}(\%)$ & $28,26 \%$ & $22,3 \%$ & 0,24 \\
Edad gestacional promedio (semanas) & $29,1 \pm 2,39$ & $29,38 \pm 2,17$ & 0,3 \\
Niños $\leq 27$ semanas (\%) & $28,2 \%$ & $21,4 \%$ & 0,17 \\
Promedio hospitalización (días) & $61,7(19-258)$ & $57,4(16-243)$ & 0,12 \\
Edad promedio de la madre (años) & $32,8 \pm 6$ & $27,1 \pm 7,33$ & $<0,001$ \\
Madres $\leq 18$ años (\%) & $1 \%$ & $15 \%$ & $<0,001$ \\
Familia monoparental (\%) & $7 \%$ & $13 \%$ & 0,09 \\
Hospitalizaciones posteriores (\%) & $43 \%$ & $32 \%$ & 0,05 \\
Más de una hospitalización (\%) & $30 \%$ & $40 \%$ & 0,17 \\
Co-morbilidades (\%) & $41,8 \%$ & $41,8 \%$ & 0,97 \\
Rango edad niño (meses)* & $5-45$ & $3-43$ & \\
\hline
\end{tabular}

*Rango edad niño al momento de encuesta telefónica

HCPUC Hospital Clínico de la Pontificia Universidad Católica de Chile. HSR Hospital Dr. Sótero del Río.

Tabla 2. Diagnósticos neonatales en prematuros de muy bajo peso en dos centros

\begin{tabular}{|lccccc|} 
Diagnósticos neonatales & \multicolumn{2}{c}{ HCPUC } & \multicolumn{2}{c}{ HSR } & Valor p \\
Sin patología & $\mathrm{n}$ & $(\%)$ & $\mathrm{n}$ & $(\%)$ & \\
Displasia broncopulmonar & 54 & $(58,2)$ & 144 & $(58,2)$ & 0,97 \\
Enterocolitis necrosante & 18 & $(19,8)$ & 42 & $(16,5)$ & 0,59 \\
Compromiso neurológico & 5 & $(5,5)$ & 6 & $(2,5)$ & 0,16 \\
Síndrome genético & 3 & $(3,3)$ & 15 & $(6,3)$ & 0,3 \\
Cardiopatía congénita compleja & 0 & $(0)$ & 1 & $(0,4)$ & 0,54 \\
Displasia broncopulmonar + compromiso neurológico & 4 & $(4,4)$ & 2 & $(0,8)$ & 0,028 \\
Dos o más patologías & 4 & $(4,4)$ & 25 & $(10,5)$ & 0,089 \\
Total & 4 & $(4,4)$ & 11 & $(4,6)$ & 0,96 \\
\hline
\end{tabular}




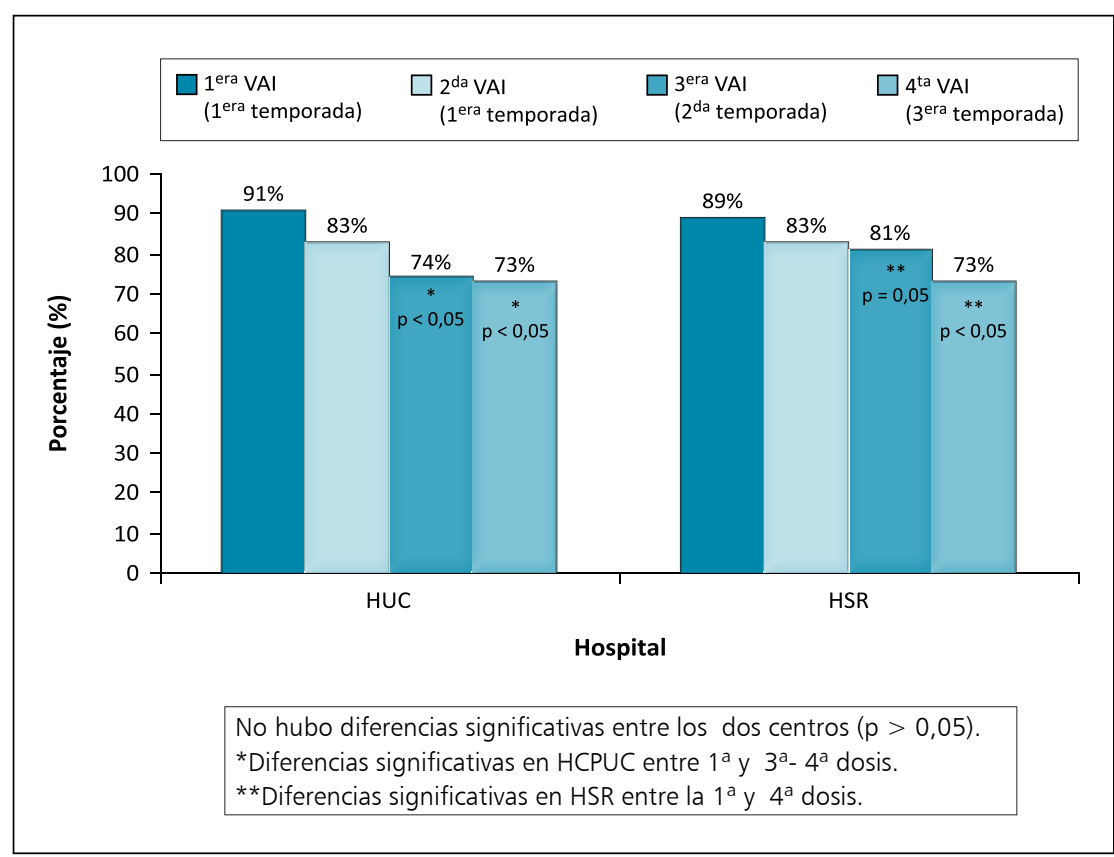

Figura 2. Proporción de prematuros con administración oportuna de dosis de vacuna anti-influenza por centro y temporada. VAl: vacuna anti-influenza.

Tabla 4. Administración oportuna del Programa Nacional de Inmunizaciones según distintas variables de los RNMBPN por centro

\begin{tabular}{|lccccc|}
\hline Variable & $\begin{array}{c}\text { HCPUC } \\
\text { \% vacunación } \\
\text { oportuna }\end{array}$ & p & $\begin{array}{c}\text { Contro } \\
\text { \% vacunación } \\
\text { oportuna }\end{array}$ & p \\
PN $\leq 1.000 \mathrm{gr}$ & 27 & 0,1 & 4 & $<0,001$ \\
EG $\leq 27$ semanas & 23 & 0,035 & 0 & $<0,001$ \\
Presencia de patología neonatal & 25 & 0,006 & 12 & $<0,001$ \\
Hospitalizaciones posteriores & 25 & 0,009 & 18 & 0,003 \\
Familia monoparental & 17 & 0,22 & 22 & 0,27 \\
Total & 40 & & 30 & 0,08 \\
\hline EG: edad gestacional & & & & \\
El valor $p$ se expresa en relación a la presencia o ausencia de la variable, para cada centro. & \\
\hline
\end{tabular}

\section{Discusión}

El desarrollo de cuidados avanzados neonatales ha permitido que prematuros, cada vez más pequeños, sobrevivan. Estos pacientes tienen mayor susceptibilidad a infecciones por inmunosupresión "funcional" explicable por inmadurez del sistema inmune, falta de anticuerpos maternos transplacentarios, escasa o nula alimentación enteral (esencial en la maduración inmunológica), ausencia del efecto protector de la leche materna, inmadurez anatómica y funcional de la vía aérea y pulmón, así como por
Tabla 3. Proporción de niños con administración oportuna del Programa Nacional de

Inmunizaciones, por vacuna, según centro

\begin{tabular}{|lccc} 
& \multicolumn{3}{c}{ Centro } \\
Vacuna/edad & HCPUC & HSR & Valor p \\
BCG* & $100 \%$ & $100 \%$ & NS \\
Penta** o Hexa*** (2 meses) & $87 \%$ & $69 \%$ & 0,1 \\
\hline Polio oral (2 meses) & $88 \%$ & $52 \%$ & $<0,001$ \\
Penta o Hexa (4 meses) & $79 \%$ & $67 \%$ & 0,03 \\
\hline Polio oral (4 meses) & $78 \%$ & $60 \%$ & 0,002 \\
Penta o Hexa (6 meses) & $69 \%$ & $62 \%$ & 0,23 \\
\hline Polio oral (6 meses) & $69 \%$ & $57 \%$ & 0,046 \\
Tresvírica****(12 meses) & $69 \%$ & $81 \%$ & 0,05 \\
\hline DPT (18 meses) & $70 \%$ & $82 \%$ & 0,08 \\
\hline
\end{tabular}

*Bacilo de Calmette y Guérin (vacuna contra la tuberculosis)

**Vacuna pentavalente (DTP/HB/Hib) contiene los siguientes antígenos: toxoide diftérico, tetánico, Bordetella pertussis de "células enteras", antígeno de superficie de virus hepatitis B más antígeno capsular de $\mathrm{H}$. influenzae tipo b conjugado)

***Vacuna hexavalente (DTPa-IPV-HB/Hib) contiene los siguientes antígenos: toxoide diftérico, tetánico, Bordetella pertussis acelular, antígeno de superficie de virus hepatitis B, virus polio 1,2 y 3 inactivado más antígeno capsular de $\mathrm{H}$ influenzae tipo b conjugado) ****Tresvírica: vacuna anti sarampión, rubéola y parotiditis.

En la tabla la vacuna polio oral se registra en forma separada pues en el HSR se usa el esquema PNI (pentavalente inyectable más OPV) pero en el HCPUC este antígeno está incluido en la vacuna hexavalente en forma de virus polio inactivado inyectable

patologías propias del prematuro. Por lo tanto, el manejo integral de estos niños requiere necesariamente del uso óptimo de medidas eficaces de prevención de infecciones entre las que se encuentra la administración de vacunas ${ }^{10}$.

El presente estudio aporta nueva información local sobre la adherencia a las vacunas de los niños de bajo peso al nacer en dos centros asistenciales. Las características biodemográficas y clínicas (Tabla 1) de los niños estudiados, son similares por lo que las escasas diferencias encontradas no son atribuibles a esto. Sí se observan diferencias en la proporción de madres muy jóvenes en el HSR, lo cual es explicable por el tipo de población más vulnerable que atiende este centro, variable que no impacta, sin embargo, en la adherencia. Existe una diferencia significativa en el porcentaje de cardiopatías congénitas en niños que nacen en el HCPUC, lo cual es atribuible a que éste es un centro de derivación nacional para cirugía cardíaca y es posible que influya en la mayor proporción de hospitalización post alta de Neonatología registrada en esta institución.

Esta serie demuestra que la vacunación oportuna ( $\sin$ atrasos) para el total de vacunas PNI en estas dos poblaciones sólo alcanza al 30-40\%, lo cual coincide con datos de 
países desarrollados que muestran cifras de $35 \%-50 \%{ }^{26-29}$. La proporción de niños vacunados oportunamente con tres dosis de DTP-HB-Hib y Polio es un indicador usado para evaluar los programas de vacunación y se considera aceptable una cifra de 90-95\%. En el HCPUC el esquema está completo en 100\% (76/76) de los casos. En cambio, en el HSR sólo $80 \%(150 / 187)$ de los niños han recibido el esquema completo debido a que se posterga la primera dosis de vacuna polio oral viva en las unidades de neonatología por el riesgo de transmisión del virus a otros niños al interior de la unidad. Este problema persiste después del alta pues en los controles ambulatorios sólo $40 \%$ de los niños recuperan la dosis de VPO no administrada. En el HCPUC se indica durante la hospitalización, regularmente, vacuna hexavalente de uso i.m., que contiene virus polio inactivado, lo que permite una alta cobertura. Si bien las implicancias de no recibir una dosis de polio son limitadas en un país que no registra casos de polio silvestre en 35 años, parece necesario considerar el uso de opciones como la vacuna hexavalente. Esta vacuna contiene, además, una formulación de antígenos de pertussis acelular que registra una menor frecuencia de reacciones adversas comunes como fiebre y otras propias del prematuro como episodios de apnea, bradicardia y desaturación ${ }^{30}$.

El atraso de las vacunas, si bien es marcado en los primeros seis meses de vida (Tabla 3), con adherencias cada vez más bajas con dosis sucesivas de DTP/HB/ $\mathrm{Hib}$, destaca que también hay atrasos importantes en la administración de las vacunas de los 12 y 18 meses (69\% para tresvírica en HCPUC) por lo tanto, en este período también es necesario reforzar el cumplimiento del calendario de vacunación.

En relación a vacuna anti-influenza, destaca un comportamiento similar para los dos centros, con una alta adherencia (89-91\%) para la primera dosis y algo menor $(83 \%)$ para la segunda dosis. Estas cifras son más elevadas que las publicadas para población de niños de término sanos y sugiere que los padres y el personal de salud perciben los riesgos asociados a la infección por virus influenza. En los años sucesivos, sin embargo, la adherencia disminuye en forma significativa (Figura 2) lo que requiere una mejor supervisión ya que los niños pequeños tienen tasas de hospitalización por complicaciones, similares a las del adulto mayor con patología crónica $^{7-8}$. Otra estrategia recomendada pero que no fue evaluada en este estudio, es el uso de vacuna anti-influenza en contactos intradomiciliarios, medida que es aún más importante en el caso de nacidos prematuros, bajo 6 meses de vida, que aún no pueden ser vacunados.

En este estudio, las razones para no inmunizar oportunamente fueron consideradas como no justificadas en una alta proporción (78\%) lo que fue similar en los dos centros. Los argumentos que los padres aducen para esto son diferentes. Una de las principales es la hospitalización por intercurrencias, situación que podría evitarse reforzando a los padres la seguridad de vacunar precozmente después de una hospitalización. Uno de los posibles factores que confunde el esquema de vacunación es la percepción de que estos niños deben ser evaluados como en el crecimiento y desarrollo, según edad corregida y no según edad cronológica, hecho que se observó en este estudio.

Una de las vacunas de gran importancia médica como es PCV7, sólo se administra en forma completa a 15,3\% de los niños en un centro privado, probablemente por limitaciones económicas y sólo a $9 \%$ de los niños en un hospital público, a pesar de que en éste la vacuna teóricamente está disponible en forma gratuita para esta población. Esto en parte se debe a problemas de stock disponible en el HSR. Puede ser subsanado con una mayor capacitación del personal de salud y asegurando una dotación suficiente de vacuna PVC7 en los hospitales públicos.

La proporción de niños MBPN vacunados adecuadamente con vacunas adicionales extra PNI es baja en ambos centros hospitalarios y muestra diferencias en el tipo de vacuna prescrita, probablemente por ponderar el pediatra la posibilidad de los padres de adquirir vacunas de alto costo. La indicación de vacuna anti-rotavirus es muy baja lo que puede deberse además del factor económico a que la información sobre su recomendación, seguridad y eficacia en prematuros es más reciente ${ }^{23}$. Parece importante que la población esté informada de la existencia de estas nuevas vacunas y eventualmente pueda tomar decisiones respecto a su administración ya que los estudios demuestran que la prescripción médica es el principal factor en la decisión del uso de vacunas ${ }^{31}$.

Al analizar, otras variables que pueden influir en la adherencia a las vacunas (Tabla 4 ) el peso de nacimiento $\leq 1.000$ gr, la edad gestacional $\leq 27$ semanas, el porcentaje de hospitalizaciones posteriores al alta en Neonatología y las patologías neonatales influyen negativamente en la adherencia. Si bien en el HCPUC el peso $\leq 1.000$ gr no mostró diferencias, esto puede deberse al número pequeño de niños, ya que al sumar a todos los $\leq$ de 1.000 gr en ambos centros, la diferencia sí es significativa; por lo tanto, en estos niños la supervisión debe ser reforzada.

Una de las limitaciones de este estudio es la alta proporción de niños que no pudieron ser incluidos por falta de registros escritos, en uno de los centros $(n=71)$. Para evaluar posibles diferencias demográficas y clínicas en esta población que pudiesen significar un sesgo, se realizó un análisis separado incluyendo los datos de peso de nacimiento, edad gestacional, patologías neonatales y duración de la hospitalización y no se demostraron diferencias significativas con el resto del grupo. Otra consideración es que las causas argumentadas por los padres para los atrasos o no inmunización tienen las limitaciones propias del uso de una anamnesis remota; no hay, sin embargo, otra forma de rescatar esta información 
y por otro lado es un método usado en numerosos estudios sobre adherencia a las vacunas.

La mejoría de la adherencia a la vacunación en RNMBPN requiere de un esfuerzo adicional por educar al personal de salud y a los padres sobre la relevancia de inmunizar oportunamente según edad cronológica, así como lo indispensable que son las dosis de refuerzo. Otras medidas que en países desarrollados han demostrado mejoría en el cumplimiento de las inmunizaciones son el uso de registros computacionales de las vacunas con acceso a información confiable, expedita y transversal, que comunique centros de atención primaria y terciaria, y que incluya, además, sistemas de recordatorio o aviso automáticos para los padres y/o encargados de la vacunación.

Finalmente, parece necesario considerar a este grupo susceptible de niños en futuras ampliaciones del esquema de inmunización actual en nuestro país. Las prioridades serían ampliar la cobertura de serotipos de $S$. pneumoniae con nuevas vacunas conjugadas así como la inclusión de vacuna anti-rotavirus, y el reemplazo de la vacuna antipertussis de "células enteras" por vacunas acelulares con un mejor perfil de seguridad.

Agradecimientos. A Dalila Pinto Ch., Técnico Paramédico, del Policlínico de Seguimiento de Prematuros del Hospital Dr. Sótero del Río, por su valiosa colaboración en la recopilación de datos.

\section{Resumen}

Los niños prematuros menores de 1.500 gr o de 32 semanas de gestación al nacer (RNMBPN) tienen mayor morbimortalidad por enfermedades infecciosas. La vacunación de estos niños es una medida esencial, la que, sin embargo, es subóptima. Objetivo. Describir la adherencia a las vacunas programáticas (PNI) y adicionales recomendadas en RNMBPN en dos centros y comparar su nivel de cumplimiento. Pacientes $y$ Métodos. Se incluyeron RNMBPN nacidos entre enero 2006 y diciembre 2008 de un centro privado (HCPUC) y otro del sistema público (HSR). Se registraron a partir de fichas neonatales y de consultorio de seguimiento, datos demográficos, diagnósticos, vacunas PNI, retrasos y causas de éstos y prescripción de vacunas adicionales. Resultados. Se describen 92 niños en el HCPUC y 246 en el HSR, 60 y 70\% respectivamente tienen alguna vacuna atrasada. En ambos centros las razones fueron no justificadas en $80 \%$ de los casos, siendo la razón principal olvido o falta de tiempo de los padres. El uso de vacunas adicionales es aún escaso en ambos centros. Conclusiones. La vacunación del prematuro en nuestro país requiere mejorías que incluyan educación del personal de salud y padres, mejoras en los registros e incorporación al programa de nuevas vacunas eficaces y seguras sin costo para los pacientes.

\section{Referencias}

1.- Plotkin S L, Plotkin S A. A short history of vaccination. Plotkin SA, Orenstein WA, Offit P A. editors. Vaccines $5^{\text {th }}$ ed. Philadelphia: WS Saunders, 2008, p. 1-16.

2.- De Arístegui J. Introducción. Asociación Española de Pediatría, Comité Asesor De Vacunas (1998-2002). Manual de vacunas en pediatría 2da ed. Latinoamericana, 2003, p. Xi-Xii.

3.- Long S S, Pickering L K, Prober C G, eds. Principles and Practice of Pediatric Infectious Diseases. New York, NY: Churchill Livingstone Inc; 1997: 596-603, 607-8, 619-25, 981.

4.- Solís Y, Bolte L, Johnson M, Cerda J, Potin M. Adherencia a las inmunizaciones en niños con necesidades de cuidado médico especial. Rev Chil Infect 2007; 24: 485-90.

5.- Langkamp DL, Davis JP. Increased risk of reported pertussis and hospitalization associated with pertussis in low birth weight children. $\mathrm{J}$ Pediat 1996; 128: 654-9.

6.- Shinefield H, Black S, Ray P, Fireman B, Shwalbe, Lewis E. Efficacy, immunogenicity and safety of heptavalent pneumococcal conjugate vaccine in low birth weight and preterm infants. Pediatr Infect Dis J 2002; 21 :
$182-6$.

7.- Neuzil K, Mellen B, Wright P, Mitchel E $\mathrm{Jr}$, Griffin M . The effect of influenza on hospitalizations, outpatient visits, and courses of antibiotics in children. N Engl J Med 2000; 342: 225-31.

8.- Izurieta $\mathrm{H}$, Thomson $\mathrm{W}$, Kramarz P, Shay D, Davis R, Destefano F, et al. Influenza and the rates of hospitalization for respiratory disease among infants and young children. N Engl J Med 2000; 27; 232-9.

9.- Batra J, Eriksen E, Zangwill K, Lee M, Marcy M, Ward JI. Evaluation of vaccine coverage for low birth weight infants during the first year of life in a large managed care population. Pediatrics 2009; 123: 951-8.

10.- Potin M, Valencia A. Vacunación del niño prematuro: Un tema a veces olvidado. Rev Chil Infect 2005; 22: 339-44.

11.- Pinquier D, Adde-Michela C, Ploin D, Levêque C, Marret S. Vaccination rate of premature infants at 6 and 24 months of age: a pilot study. Arch Pediatr 2009; 16: 1533-9.

12.- Saari T. American Academy of Pediatrics. Committee on Infectious Diseases. Immunization of preterm and low birth weight infants. Pediatrics 2003; 112: 193-8.

13.- Advisory Committee on Immunization
Practices. (2005). A comprehensive immunization strategy to eliminate transmission of hepatitis B virus infection in the United States. Part 1: Immunization of infants, children, and adolescents. MMWR Morb Mortal Wkly Rep 54(RR-16), 1-31.

14.- Advisory Committee on Immunization Practices. (2006a). Preventing tetanus, diphtheria, and pertussis among adolescents: Use of tetanus toxoid, reduced diphtheria toxoid and acelular pertussis vaccines. MMWR Morb Mortal Wkly Rep 55 (RR-3), 1-34.

15.- Advisory Committee on Immunization Practices. (2006b). Preventing tetanus, diphtheria, and pertussis among adults: Use of tetanus toxoid, reduced diphtheria toxoid and acellular pertussis vaccine recommendations of the Advisory Committee on Immunization Practices (ACIP) and recommendation of ACIP, supported by the Healthcare Infection Control Practices Advisory Committee (HICPAC), for use of Tdap among health-care personnel. MMWR Morb Mortal Wkly Rep 55 (RR- 17): 1-37.

16.- Advisory Committee on Immunization Practices. (2006c). Prevention and control of influenza. MMWR Morb Mortal Wkly Rep 55 (RR-10), 1-42. 
17.- Advisory Committee on Immunization Practices. (2006d). Prevention of rotavirus gastroenteritis among infants and children. MMWR Morb Mortal Wkly Rep 55 (RR-12), 1-13.

18.- Davis R, Rubanowice D, Shinefield H, Lewis N, Gu D, Black S, et al. Immunization levels among premature and low-birth-weight infants and risk factors for delayed up-to-date immunization status. JAMA. 1999; 282: 547-53.

19.- Moyes C. Immunization of preterm babies. N Z Med J 1999; 112: 263-4.

20.- Slack M H, Schapira D. Severe apneas following immunizations in premature infants. Arch Dis Child Fetal Neonatal Ed. 1999; 81: F67-8.

21- Kirmani K, Lofthus G, Pichichero M, Voloshen T, D‘Angio C, Seven year follow-up of vaccine response in extremely premature infants; Pediatrics 2002; 109: 498-504.
22.- Registro estadístico del Servicio de Neonatología del Hospital Clínico Pontificia Universidad Católica de Chile. 2006-2008.

23.- Registro estadístico del Servicio Asistencial Dr. Sótero del Río. Servicio de Neonatología. 2006-2008.

24.- Varrasso D A, Redlener I. Defining delayed immunizations. Pediatr Infect Dis J 1992; 11: 897.

25.- Dietz V J, Zell E R, Stevenson J. Defining delayed immunization. Pediatr Infect Dis J 1993; 12: 353-4.

26.- Bonhoeffer J, Siegrist C, Heath P. Immunisation of premature infants. Arch Dis Child 2006; 91; 929-35.

27.- Ashraf G, Shah S. Special immunization considerations of the preterm infant. J Pediatr Health Care 2007; 21: 385-91.

28.- Gaudelus J, Lefèvre-Akriche S, Roumegoux
C, Bolie S, Belasco C, Letamendia-Richard E, et al. Immunization of the preterm infant. Arch Pediatr 2007; 14: 24-30.

29.- Esposito S, Serra D, Gualtieri L, Cesati L, Principi N. Vaccines and preterm neonates: Why, when, and with what. Early Human Development 2009; 85: 43-5.

30.- Schloesser R, Fisher D, Otto W, RettwitzVolk W, Herden P, Zielen S. Safety and immunogenicity of an acellular pertussis vaccine in premature infants. Pediatrics 1999; 103: e60.

31.- Taylor J, Darden P, Slora E, Hasemeier C, Asmussen L, Wasserman R. The influence of provider behavior, parental characteristics and public policy initiative on the immunization status of children followed by private pediatricians: a study from pediatric research in private office settings. Pediatrics 1997; 99: 209-15. 